\title{
Shape correction in transient thermography inspection of non-planar components
}

\author{
by A. NOUAH, X. MALDAGUE and F. ROBITAILLE
}

Laboratoire de Vision et Systèmes Numériques, Département de Génie électrique, Université Laval, Québec, QC, Canada, G1K $7 P 4$.

Abstract

Transient thermography is a very attractive non destructive evaluation tool however; despite recent advances, some difficulties still remain. One important concern is the inspection of non planar components for which the required external thermal stimulation produces isotherm disparities perturbing the interpretation stage. In this paper a novel method is proposed to solve this problem. The method is based on depth correction using the reflectance information obtained from a visible spectrum camera. Theory is discussed and results are presented.

\section{Introduction}

Transient thermography (TT) is now emerging as a mature non destructive evaluation (NDE) technique. One of its most important field of application is for aerospace components having often non planar shapes. In the traditional TT inspection in reflection, the surface of the component is briefly thermally stimulated and the recorded evolution of surface temperature witnesses presence of subsurface flaws $[1,2,3]$.

This ideal inspection scheme is afflicted by several difficulties such as the necessity for a known/ high surface emissivity $\varepsilon$ and also the necessity to uniformly stimulate the inspected surface. Moreover, if non-planar surfaces are inspected by TT, new problems arise due to local overheating caused by specimen curvature. These local overheating areas perturbs the image interpretation step and can be even falsely misinterpreted as subsurface flaws.

They are several ways to overcome this problem of inspection of non planar components: tridimensional infrared ranging is now slowly emerging and has been already experimentally demonstrated although depth resolution is gross and much developments are still needed [4]. Another possible approach is to combine together infrared and visible data obtained from the inspected object. Fusion of sensory information has been already proposed, see $[5,6]$ for instance but in a different context, mainly to ease outdoor scenes interpretation and object recognition. The approach we propose here is dedicated to NDE and the data processing is novel. The main stream of information is the timely temperature evolution of the specimen while the visible information is only used for curvature correction, to flatten shaped objects since visible reflectance data allows to estimate local orientation of specimen surface.

This research effort opens new possibilities in term of detection and quantitative evaluation of subsurface flaws in the case of TT inspection of non planar components. In the paper, the fundamental theory of this novel approach will be discussed and examples of experimental investigations will be given.

\section{Theoretical aspects}

The temperature increases $d T\left[{ }^{\circ} \mathrm{C}\right]$ of a surface patch $d A_{x y}$ submitted to thermal stimulation by a source of $P\left[\mathrm{~W} / \mathrm{m}^{2}\right]$ located at a distance $R$ from the surface can be expressed, for short time intervals, by $[7$, eq. $3-40]$ :

$$
d T \approx \frac{\varepsilon P \cos \theta d t}{\rho C_{p} d z k_{1} R^{2}}
$$

with: $\quad d t$ time at which the surface is observed [s], 


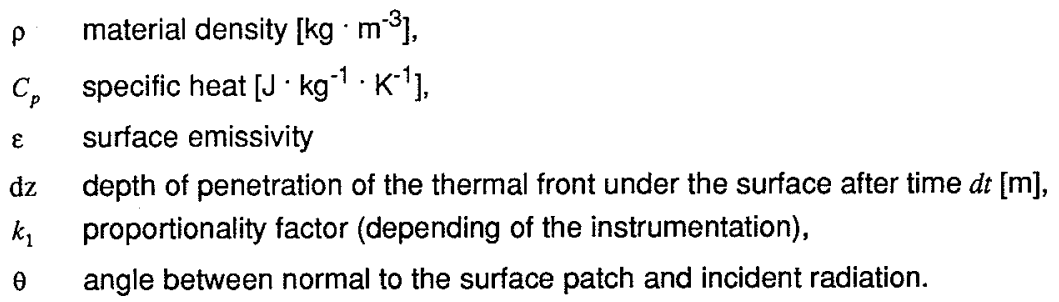

This relation indicates the various parameters which influence temperature rise on the specimen surface. On particular interest are: surface orientation $\theta$ and distance $R$. If the stimulation source is large with respect to the specimen size, as it is generally the case in NDE applications, the influence of distance $R$ can be neglected. However, unless the inspected specimen is flat and lies perpendicularly to the stimulation source, surface orientation $\theta$ has to be considered. This is the case for the inspection of non planar components. If surface orientation $\theta$ can be determined, then its effect can be eliminated in equation (1) and the thermal inspection can proceed as in the case of flat components.

A possible approach to determine $\cos \theta$ is to make use of the reflectance information of the scene present in a visible image. Many different methods have been proposed in the literature to extract surface orientation such as for instance stereoscopy [8], laser ranging [9] or the use of shading to extract the shape [10]. These methods are relatively complex to use requiring either a known alignment of the cameras, an active measuring scheme, or they impose severe restrictions on both the object and lighting. NANDHAKUMAR et al. [5] proposed a simple direct method to extract $\cos \theta$ from a visible image. This method assumes that observed surfaces are Lambertian and objects opaque. These hypotheses are valid for our particular application. In this conditions:

$$
L=K \cos \theta+C
$$

where: $\quad L$ is the digitized value of the intensity of the visible image,

$K$ and $C$ are the overall gain and offset of the visible imaging system.

Both $K$ and $C$ are fixed for a given camera calibration (optics aperture, system gain and black level offset), they can be found experimentally using the following procedure. An image of a scene with two surfaces of known orientation is digitized. Substitution of known orientations and digitized values for the two surfaces in equation (2) allows to write a two-equation system which solves for $K$ and $C$. This method can be used to compute elementary surface orientation at the pixel level although strictly speaking the modulation transfer function (MTF) should be taken into account. This is however not especially a problem in this application where spatial resolution of visible images are higher than for infrared images.

In order to compare them, the two images (visible and infrared) must be registered. This is essential if we want a point on the inspected surface to have the same coordinates in the two images. This step in the correction procedure must be taken into account since image formats are different for both cameras. Registration can be done using the following transformation between the coordinates $(x, y)$ and $(u, v)$ for infrared and visible images respectively. For the $u$ coordinate we can write:

$$
u=\sum_{i=0}^{N} \sum_{j=0}^{N-i} a_{i j} x^{i} y^{j}
$$

with: $\quad \mathrm{N}$ degree of the polynomial (usually 2),

$a_{i j}$ polynomial coefficients.

The $v$ coordinate is computed using a similar relationship. In order to establish the polynomial coefficients, a set of common points between two images of the same scene, visible and infrared, are selected (either manually or with a pairing program) and a system of equations is solved. Obviously, these computed coordinates are not necessary under an integer format and consequently interpolation between coordinates is necessary to obtain image values at the exact integral coordinates. An another important point is the background which is easily eliminated in 


\section{http://dx.doi.org/10.21611/qirt.1992.034}

this procedure by taking into consideration only the object points having a strong thermal contrast due to the heating pulse (background is located much further back and is not much affected by the heating device). Using this method, points in the two images can be compared.

The last step for the shape correction of thermograms consists to multiply thermograms by inverse of the orientation $(1 / \cos \theta)$, recalling that orientation is extracted from the visible image equation (2). Ideally, following equation (1), this last operation should correct for shape curvature of the objects. However, many adverse conditions can reduce efficiency of the method. For instance, equation (2) is only valid if lighting for the visible camera is made up of parallel rays (see \$4). Another important point to consider is the uniform thermal stimulation deposition made by the heating device (non uniformities will perturb results as we will see below).

\section{Experimental configuration}

Figure 1 shows the experimental set-up. An infrared (Inframetrics 600, 8-12 $\mu \mathrm{m}$ ) and a visible camera (Panasonic WV-CD 50, $0.4-1.1 \mu \mathrm{m}$ ) are mounted with their optical axis being parallel in front of the object to inspect. The inspected object is mounted on an optical bench and positioned to fall entirely in the field of view shared by both cameras. Two $1000 \mathrm{~W}$-back reflector projectors are used to thermally stimulate the component. A lamp illuminates the scene (a reduced power $60 \mathrm{~W}$-back reflector projector was used because the $1000 \mathrm{~W}$ heating projectors saturated the visible camera). The acquisition station of the lab is used to control the set-up, record and process the images [11].

After the calibration procedure exposed in $\S 2$ has been performed using equations $(2,3)$, the experimental procedure for shape correction consists first to proceed to the traditional thermographic inspection scheme (flash heat the specimen and record subsequent surface temperature response for possible anomaly witnessing presence of subsurface flaws), the visible image is next recorded. This order (infrared recording first) prevents possible adverse heating of the specimen which would disturb the subsequent data analysis (e.g. inversion procedure or comparison with a thermal modelling of the specimen behaviour).

\section{Results and discussion}

Figures 2, $A^{*} B$ present some results recorded over a $15 \mathrm{~cm}$ diameter plastic half sphere. Although truly artificial, this particular specimen was chosen since it exhibit surface orientation from 0 to $90^{\circ}$ while plastic can be easily machined. A $5 \mathrm{~mm}$ diameter hole was drilled from the back surface being $1.2 \mathrm{~mm}$ below the front (inspected) surface. In order to increase the plastic emissivity, the specimen was covered with a Tremco' gray flat paint which has both good pseudo-Lambertian properties in the visible spectrum and an acceptable emissivity value in the infrared band of our imager ( 0.7).

The visible image of the half sphere is on figure 2. Figure $A$ shows the infrared image (a) before and (b) after the registration operation of $\$ 2$ (recorded at time of maximum thermal contrast over the defect, some $6 \mathrm{~s}$ after heating for $3 \mathrm{~s}$ ). The shape-corrected image is displayed on figure $B$. Profiles of a row passing over both defect and sphere center are shown on figure 3, (a) before (extracted from figure $A-b$ ) and $(b)$ after shape correction (same row number, extracted from figure $B$ ). On figures $A$ and 3-a, distortions due to shape are well observed, up to the point that visibility of the subsurface flaw is significantly reduced. On the contrary, the corrected image (figure $B$ and profile of figure 3-b) exhibits a much enhanced defect contrast. On figure $B$, some edge effects are observed, this is caused by the strong $90^{\circ}$ curvature of the sample contour for which no reliable information is available. Also noticed on figure $B$ are some non uniform temperature areas caused by the uneven heating by the two overlapping thermal pattern of the $1000 \mathrm{~W}$ projectors. Finally, notice than on figures $A, B, 3$, the infrared image was registered with the visible image; it is better to do the opposite in order to avoid size distortion of relevant features (observed subsurface defect) in the thermograms.

An important aspect to consider in order to apply the proposed procedure efficiently is to rely on a parallel-ray lighting source for the visible image (such as a back reflector projector or a bank of fluorescent tubes). This is very important because if, say a point source is used (such as using an omni-directional incandescent light bulb), the illumination obtained will vary as $I / R^{2}$ (where $R$ is the source-object distance). In this case the correction process is no longer straightforward. Figure 4 illustrates the problem, a tilted plane $\left(35^{\circ}\right.$ between normal to the surface and the optical axis of the camera) is observed with the visible camera while illuminated by a $60 \mathrm{~W}$ incandescent light bulb. Although the orientation is constant on the plane surface, the profile of figure 4 indicates a definitive trend due to variation of distance between light source and observed surface patches.

A limitation of the procedure is that the maximum depth of the object should be smaller than the

* The colour plates of this article 34 are located on page XI of the colour gathering, at the end of the book 
depth of focus of the cameras. However, since the field of view is some what restricted to about $20 \times 20 \mathrm{~cm}^{2}$ in TT investigations, we did not found this limitation to be too severe but to inspect very awkward objects.

Another point is the emissivity $\varepsilon$ which, for many materials, is a function of the orientation $\varepsilon(\theta)$. Generally, for specular surfaces (e.g. polished metal surfaces), $\varepsilon$ is relatively constant up to $40^{\circ}$, Increases slightly from 40 to $80^{\circ}$ before to drop for higher orientations. For flat bodies, $\varepsilon$ is constant up to about $60^{\circ}$ [12]. This implies the validity of the proposed method (without further consideration for the emissivity) has thus a restricted orientation span. This is another explanation for the non uniformities observed on the contour (high orientation) of the shape corrected object (figure $B$ ). Of course, if the orientation behaviour of the emissivity is known, it is possible to take its effect into consideration in the shape correction procedure and then increase the validity span.

The shape correction procedure described in this paper appears well effective and allows to process thermograms of non planar objects which could otherwise hardly be handled by procedures such as quantitative evaluation [13], thermal diffusivity measurement [14] or thermal tomography processing [15]. This novel method flatten temperature response distribution leading to a better defect visibility and improved analysis. Work is still needed to ameliorate the usefulness of the method, e.g. more uniform heating, emissivity analysis. Work in this direction is in progress.

\section{Acknowledgments}

The financial support of the Natural Sciences and Engineering Research Council of Canada (NSERCC) and of the Fonds pour la Formation de Chercheurs et l'Aide à la Recherche (FCAR) of the Québec's Province is acknowledged.

\section{REFERENCES}

[1] MALDAGUE (X.). - Nondestructive evaluation of materials by infrared thermography. London, Springer-Verlag, 296 p., 1992 (in-press).

[2] LAU (S.K.), ALMOND (D.P.) and MILNE (J.M.). - A quantitative analysis of pulsed video thermography. NDT \&E, 24 [4], Aug. 1991, p. 195-202.

[3] BALAGEAS (D.) - Le contrôle non destructif par méthodes thermiques. Rev. Gén. Therm. Fr., $\mathrm{n}^{\circ}$ 356-357, Aug-Sept. 1991, p. 483-498. [in French]

[4] MALDAGUE (X.), FORTIN (L.) and PICARD (J.). - Applications of tridimensional heat calibration to a thermographic NDE station. Baird (G.S.) ed., Thermosense XIII, SPIE Proc. 1467, 1991, p. 239-251.

[5] NANDHAKUMAR (N.) and AGGARWAL (J.K.) - Integrated analysis of thermal and visual images for scene interpretation. Trans. IEEE, PAMi-10 [4], July 1988, p. 469-481.

[6] DUANE (G.). - Pixel-level sensor fusion for improved object recognition. Weaver (C.B.) ed., Sensor fusion, SPIE Proc. 931, 1988, p. 180-185.

[7] THOMAS (L.C.). - Fundamentals of heat transfer. Englewood Cliffs: Prentice-Hall, 435 p., 1980.

[8] KAMGAR-PARSI (B.) and EASTMAN (R.D.). - Calibration of a stereo system with small relative angles. Comput. vis., gr. \& imag. proc., 51 [1], July 1990, p. 1-19.

[9] BLAIS (F.), RIOUX (M.) and BERALDIN (J.A.). - Practical considerations for the design of a high precision 3-D laser scanner system. Optomechanical and electro-optical design of industrial systems, SPIE Proc. 959, 1988, p. 225-246.

[10] BRUCKSTEIN (A.M.). - On shape from shading. Comput. vis., gr. \& imag. proc.,44 [2], Nov. 1988 , p. 139-154.

[11] ROBITAILLE (F.) and MALDAGUE (X.). - Infrared thermographic inspection station: experimental set-up and working environment. Bogdady (G.) ed., Broc. Industrial Automation Conf., Montréal, June 1-3, 1992, p. 22.5-22.7.

[12] SPARROW (E.M.) and CHESS (R.D.). - Radiation heat transfer. New York: McGraw-Hill, section 2-2, 1978.

[13] KRAPEZ (J.-C.), MALDAGUE (X.) and CIELO (P.). - Thermographic non destructive evaluation: data inversion procedures. Part $11: 2-D$ analysis and experimental results. Res. in NDE, 3 [2], 1991, p. 101-124. 
[14] HEATH (D.M.) and WINFREE (W.P.). - Thermal diffusivity measurement in carbon-carbon composites. Thompson (D.O.) and Chimenti (D.E.) eds., Review of Progress in Quantitative NDE, Plenum Press, 8B, 1989, p. 1613-1619.

[15] VAVILOV (V.), BISON (P.G.), BRESSAN (C.), GRINZATO (E.) and MARINETTI (S.). - Some new ideas in dynamic thermal tomography. Balageas (D.) ed., Proc. Quantitative infrared thermography, Eurotherm Seminar 27, 6 p., Paris, July 7-9, 1992.

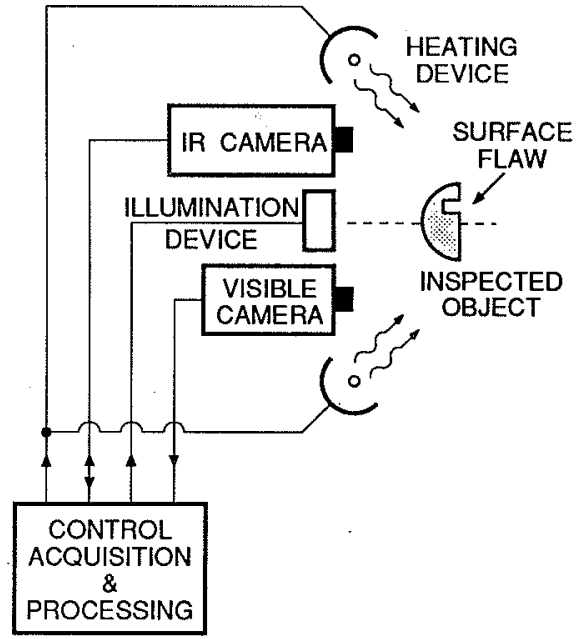

Fig. 1. - Experimental configuration.

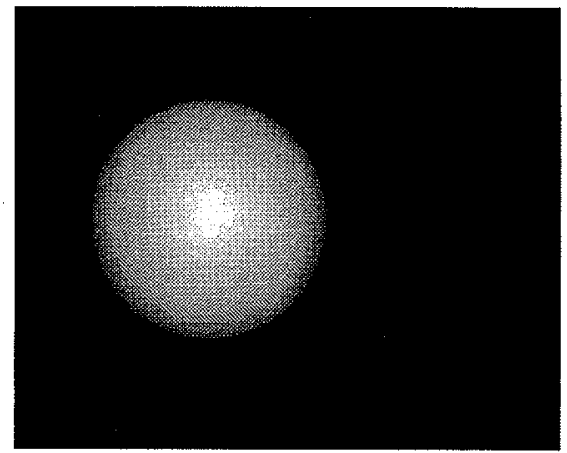

Fig. 2. - Visible image recorded over a plastic half-sphere.

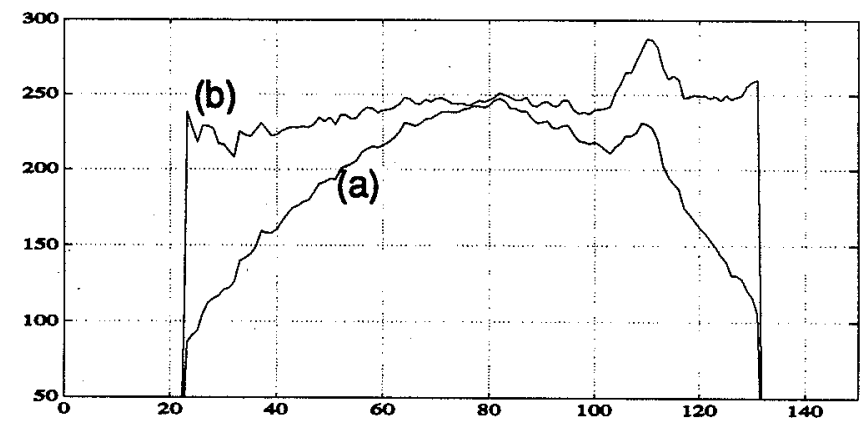

Fig. 3. - Half-sphere, profiles passing by both defect and specimen center, (a) before (Fig. A-b) and (b) after (Fig. B) shape correction, (arbitrary vertic. units, horiz. scale: pixel coordinates).

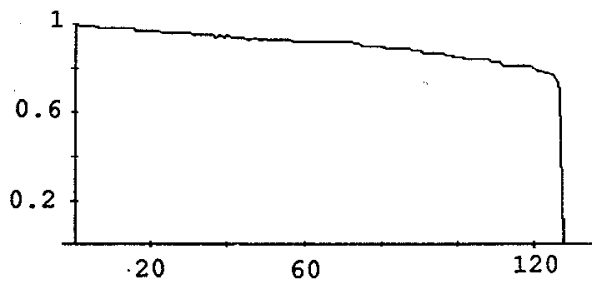

Fig. 4. - Tilted plate with point source illumination, profile of the gray level across the image: effect of $1 / R^{2}$ (arbitrary vertic. units, horiz. scale: pixel coordinates). 